\title{
Identification of potential strain heterogeneities during wetting-induced compaction
}

\author{
Constance Mihalache ${ }^{1}$ and Giuseppe Buscarnera ${ }^{1, a}$ \\ ${ }^{1}$ Northwestern University, Evanston, IL 60208, United States
}

\begin{abstract}
Compaction upon wetting has been reported to occur in various types of unsaturated soils with damaging consequences for infrastructure in field applications. This deformation process is often referred to as "wettingcollapse", implying that it may be unstable in nature. Recent evidences also indicate that compaction localization represents a possible mode of deformation during wetting, even in the presence of oedometric loading paths. Despite this, little work has been done from a mechanistic perspective to assess the susceptibility of these processes to localization instability. Here we assess the potential for strain localization during wetting through controllability analyses defined in light of a second-order work expression for unsaturated soils. A hydro-mechanical constitutive model with suction-dependent hardening is used to simulate classic experimental data, and the controllability criteria are specialized to capture the potential for shear band formation for a range of band inclinations under water contentcontrolled and suction-controlled wetting paths. The effect of changes in material characteristics was evaluated, showing that the potential for strain localization upon water-injection increases with increasing values of suctioninduced hardening, and that non-associativity may have an effect on both the potential for localization under rapid wetting, as well as on the range of band angles over which it may occur. Specifically, it is possible to distinguish two well-defined stress regions, one within which strain localization is first possible with horizontal band inclinations and another in which inclined localization zones tend to be more critical. Such results provide insight on the factors that may contribute to strain localization during wetting and find general applicability in the interpretation of the response of geo-structures subjected to intense hydrologic forcing.
\end{abstract}

\section{Introduction}

A classical problem of unsaturated soil mechanics is the study of compaction in soils subjected to wetting paths. In earthen systems, this compaction can be significant, causing substantial settlements in infrastructures such as dams and levees [1,2], as well as damage to structures [3] and transportation systems [4]. Due to its destructive nature, this phenomenon has been referred to as "wettinginduced collapse", which suggests that these events can be mechanically unstable. However, recent studies [5] inspecting the loss of wetting control in soils modelled with an elastoplastic constitutive law [6] have shown that both oedometric and isotropic saturation paths were controllable upon suction removal and water injection for the unsaturated soils considered in the study. To identify properties that may promote unstable wetting, the model parameters dictating the suction-induced hardening of the material were then increased to values higher than those indicated by the experiments. This analysis demonstrated the crucial effect of hydro-mechanical couplings on the onset of instabilities, suggesting that wetting paths may provoke unstable compaction only in soils characterized by significant water-sensitivity.

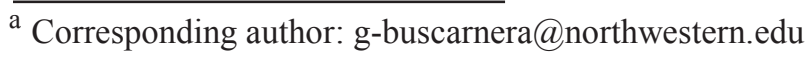

Such analyses, however, address only homogeneous modes of instability, and do not consider the potential for heterogeneous modes of deformation such as shear and/or compaction bands. Indeed, while oedometric paths imply homogenous uniaxial strains, recent experiments have identified zones of strain heterogeneity for wetting tests conducted in an oedometer [7]. Thus, it is necessary to consider the potential for strain localization also during this common class of saturation events.

While strain localization can be studied through a classic acoustic tensor approach [8,9], it is also possible to use controllability methods [10] to study such modes of instability under drained or undrained conditions [11]. In this paper the latter approach is used to assess strain heterogeneities during wetting on partially saturated soils, plotting controllability indices for wetting paths driven by fluid pressurization and/or water injection for a range of band angles. In addition, the role of various soil constants on the evolution of these indices has been inspected. In particular, it is shown that the predicted mode of instability is affected not only by the stress state, but also by suction-hardening and degree of non-associativity. In fact, depending on the considered parameters, different cases are identified where either horizontal (compactive) or inclined (shear) strain localization bands are favoured. 


\section{Controllability criteria for shear and/or compaction strain localization}

Similar to the analysis performed in [12], a material point is considered susceptible to shear localization at a certain inclination, $\theta$ (Figure 1), if it can no longer control an increment of shear stress acting along that inclination. Therefore, in addition to the axial and radial directions used in [5], we will also consider the role of shearing components perpendicular to the axial direction (Figure 1) in our controllability analysis. Consider a constitutive expression of the form,

$$
\left\{\begin{array}{c}
\dot{\sigma}_{A}^{*} \\
\dot{\sigma}_{R}^{*} \\
\dot{\tau} \\
n \dot{s}
\end{array}\right\}=\left[\begin{array}{cccc}
D_{A A} & D_{A R} & D_{A S} & D_{A H} \\
D_{R A} & D_{R R} & D_{R S} & D_{R H} \\
D_{S A} & D_{S R} & D_{S S} & D_{S H} \\
D_{H A} & D_{H R} & D_{H S} & D_{H H}
\end{array}\right]\left\{\begin{array}{c}
\dot{\varepsilon}_{A} \\
\dot{\varepsilon}_{R} \\
\dot{\gamma} \\
-\dot{S}_{r}
\end{array}\right\}
$$

where $\tau$ is shear stress, $\gamma$ is shear strain, $n$ is porosity, $s$ is suction, and the $D_{i j}$ terms are partitions of the constitutive stiffness matrix, $\boldsymbol{D}$. Variables $\sigma_{A}^{*}$ and $\sigma_{R}^{*}$ are the axial and radial stresses work-conjugate to the axial and radial strains $\left(\varepsilon_{A}\right.$ and $\left.\varepsilon_{R}\right)$, as defined by the unsaturated second-order work expression [13], or specifically,

$$
\dot{\sigma}^{*}=\dot{\sigma}-S_{r} \dot{u}_{w}-\left(1-S_{r}\right) \dot{u}_{a}
$$

where $\sigma$ is the total stress, $u_{w}$ the water pressure, $u_{a}$ the air pressure, and a superimposed dot indicates the increment of the respective variables. For the sake of simplicity, we focus on the case in which only the degree of saturation affects the mechanical response in the plastic regime, but strains do not affect the retention response (i.e., one-way hydro-mechanical coupling, or $\left.D_{H A}=D_{H R}=D_{H S}=0\right)$. The components of (1) can also be rearranged such that the constitutive expression is written in terms of net stresses $\left(\sigma^{n e t}\right)$ and water ratio $\left(e_{w}\right.$ or the product of void ratio and degree of saturation).

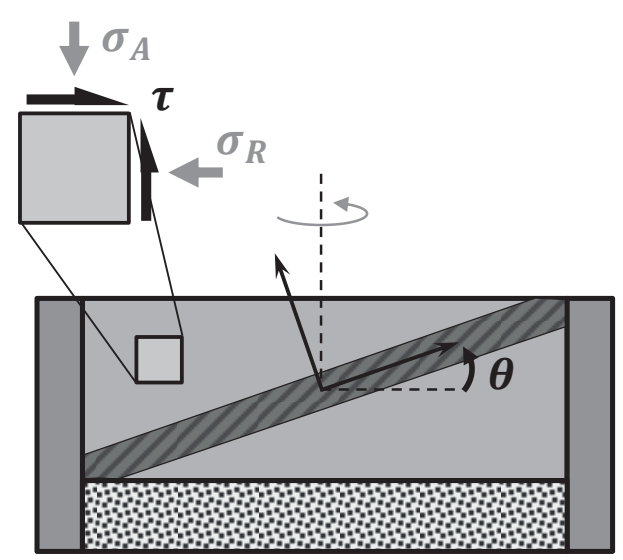

Figure 1. Schematic indicating the axis convention for the controllability analysis and the direction of shear localization

Using the loss of controllability approach [10], under the control of axial net stress, radial strain, shear stress and suction, the criterion for suction-controlled wetting becomes,

$$
\operatorname{det}\left(X_{L O C}^{S}\right)=D_{H H}\left(D_{A A} D_{S S}-D_{A S} D_{S A}\right)=0
$$

Under fulfillment of this criterion (during wetting under fluid pressurization) the material would not be able to control an increment of shear stress acting perpendicular to the axial direction. A similar criterion can also be obtained for wetting under fluid injection or water content control. In this case, the water ratio $\left(e_{w}\right)$ is controlled in addition to the axial net stress, radial strain and shear stress, and the resulting criterion is,

$$
\begin{aligned}
& \operatorname{det}\left(X_{L O C}^{W C}\right)=D_{A A}^{*} D_{S S}-D_{A S} D_{S A}^{*}=0 \\
& D_{A A}^{*}=D_{A A}-\left(D_{A H}-D_{H H} \frac{S_{r}}{n}\right) \frac{S_{r}}{n} \\
& D_{S A}^{*}=D_{S A}-D_{S H} \frac{S_{r}}{n}
\end{aligned}
$$

From the reasoning presented in [12], the formation of a deformation band at inclination, $\theta$ would require a stress perturbation parallel to the band direction. Thus, (3) and (4) would only be able to predict localization at a horizontal orientation (i.e., shear/compaction banding for the wetting-induced plastic processes considered in this work). To also consider the potential for localization under other band inclinations, the reference system must be rotated by an angle, $\theta$ so that the stress perturbation is oriented along the considered direction of potential strain localization (Figure 1). To this effect, a directional cosine rotation matrix for angle $\theta\left(\boldsymbol{T}_{\boldsymbol{R}}\right)$ can be applied to the stiffness, $\boldsymbol{D}$ [12] such that,

$$
D_{R}=T_{R} D \tilde{T}_{R}
$$

where the stiffness components in (3) and (4) can be replaced by their rotated counterparts. By computing $\boldsymbol{T}_{\boldsymbol{R}}$ over a full range of localization angles $\left(\theta=-90^{\circ}\right.$ to $\left.90^{\circ}\right)$, the potential for heterogeneity due to shear localization at different rotations can be comprehensively examined. The results can also be compared to those for wetting under homogenous deformation ( $\operatorname{det} X^{S}, \operatorname{det} X^{W C}$ in [5]).

Computation of these controllability indices requires the definition of stiffness, $\boldsymbol{D}$ via a constitutive model capable of reproducing wetting-induced compaction. In this work the elastoplastic constitutive model for unsaturated soils proposed by Buscarnera and Nova [6] is used. The model includes a hyperelastic law for the elasticity component, the ability to independently change the yield surface and plastic potential shape (i.e., for modelling non-associative materials), and a suctiondependent hardening law defined as,

$$
\dot{p}_{s}=\frac{1}{B_{p}} p_{s}\left(\dot{\varepsilon}_{v}^{p}+\xi_{s} \dot{\varepsilon}_{s}^{p}\right)-r_{s w} p_{s} \dot{S}_{r}
$$

where $p_{s}$ is the yield surface size, and $\varepsilon_{v}^{p}$ and $\varepsilon_{s}^{p}$ are the volumetric and shear components of the plastic strain. 
The parameter $r_{s w}$ controls the contraction of the yield surface that would occur during saturation (i.e., when the suction increment is negative). Additional information on the model can be found in Buscarnera and Nova [6], and the specific parameters used in this study have been calibrated for the behaviour of a sandy clay [5]. The parameter values and descriptions are given in Table 1 .

Table 1. Constitutive model parameters for sandy clay.

\begin{tabular}{|c|c|c|}
\hline Description & Symbol & Value \\
\hline Elastic Constants & $G_{0}$ & 0.008 \\
$p_{r}$ & $13000 \mathrm{kPa}$ \\
\hline $\begin{array}{c}\text { Logarithmic Plastic } \\
\text { Compressibility }\end{array}$ & $B_{p}$ & 0.045 \\
\hline $\begin{array}{c}\text { Yield Surface } \\
\text { Shape Parameters }\end{array}$ & $m_{f}$ & 0.63 \\
$M_{c}$ & 1.23 \\
\hline $\begin{array}{c}\text { Suction-Hardening } \\
\text { Parameter }\end{array}$ & $M_{e}$ & 1.10 \\
\hline $\begin{array}{c}\text { Dilatancy Parameter } \\
\xi_{s}\end{array}$ & $\xi_{v g}$ & 0.99 \\
\hline $\begin{array}{c}\text { Parameters } \\
\text { SWCC Shape }\end{array}$ & $m_{v g}$ & 0.053 \\
$n_{v g}$ & 5.6 \\
\hline
\end{tabular}

\section{Potential for heterogeneity during oedometeric wetting}

A drying, loading and wetting simulation under oedometric conditions was performed using the parameters in Table 1 . The initially saturated material at low confinement was dried under constant net stress to $45 \%$ degree of saturation, then compressed under constant radial strain to a deviatoric stress around $210 \mathrm{kPa}$, and then wet under oedometric conditions at constant axial net stress. The resulting stress path is shown in Figure 2, with a thin solid line indicating the final stress ratio reached during the simulation.

\subsection{Material parameter calibration}

The controllability indices for shear localization under suction-controlled wetting (3) and water contentcontrolled wetting (4) were monitored during the saturation path shown in Figure 2. The values for $\theta=0^{\circ}$ are plotted against the degree of saturation in Figure $3 a$, and the role of the angle of shear rotation is shown in Figures $3 \mathrm{~b}$ and $3 \mathrm{c}$ at select points along the saturation path. The indices maintain positive values throughout the wetting simulation, implying that localized instabilities are not expected during this particular wetting-induced compaction process. This indicates that the material considered in this analysis is not prone to strain localization along the path imposed by oedometric wetting. As a result, its deformation response is expected to be homogeneous, thus corroborating earlier hypotheses of homogeneity of the wetting response used by Mihalache and Buscarnera [5] to perform their analyses.

Nevertheless, it is worth noting that the indices do change in value with rotation angle, suggesting that the inclination of shearing may affect the stability of the system under other conditions. The minimum values of both criteria also occur at relatively high values of degree of saturation, which may correspond with quasi-saturated conditions in which the air phase forms occluded bubbles within the pore fluid. For the sake of simplicity, hereafter we focus on strain localization analyses for degrees of saturation smaller than $75 \%$, thus focusing only on states of saturation at which the two fluid phases can both be assumed to be continuously distributed across the sample.

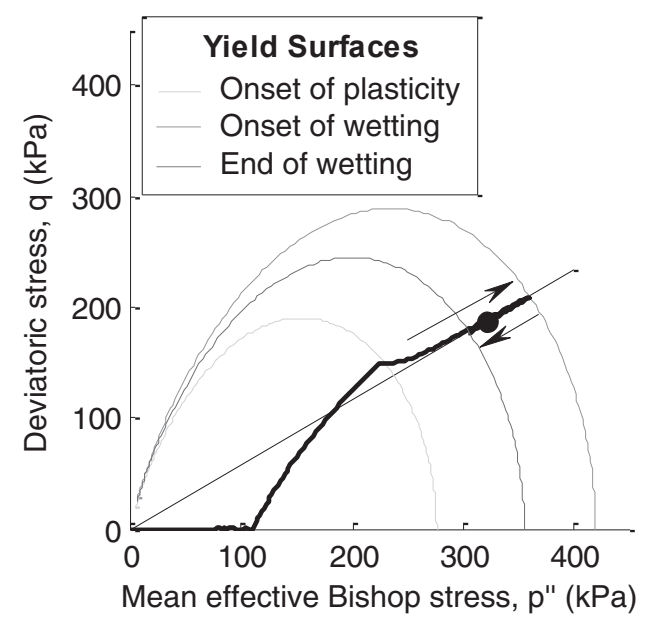

Figure 2. Oedometric stress path (thick line) with arrows indicating direction of loading and wetting. Yield surfaces for the onset of plasticity, onset of wetting and end of wetting are also shown. The point indicates the stress at which $S_{r}=75 \%$

\subsection{Role of stress ratio during wetting}

In addition to computing indices for the stress path simulated in Figure 2, it is also possible to observe how the indices vary at different stress ratios, for the same degree of saturation and along the same yield surface. Figure 4 shows the effect of considering stress ratios approximately $30 \%$ higher and $30 \%$ lower than the previous simulation. Though again all criteria remain positive throughout (so no instability is predicted at the given saturation state), the trends in the variation of the controllability indices with rotation angle do change. While for the initial simulation and for the simulation at a $30 \%$ lower stress ratio both criteria take minimum values at a horizontal band orientation, for the higher stress ratio the minimum values occur for non-zero band inclinations. The differences between the three situations suggest that the stress state at which wetting is imposed has a crucial role on the behaviour of both indices, similar to findings 
in the localization of rocks, where compaction bands and shear bands are expected at different stress ratios [14,15].
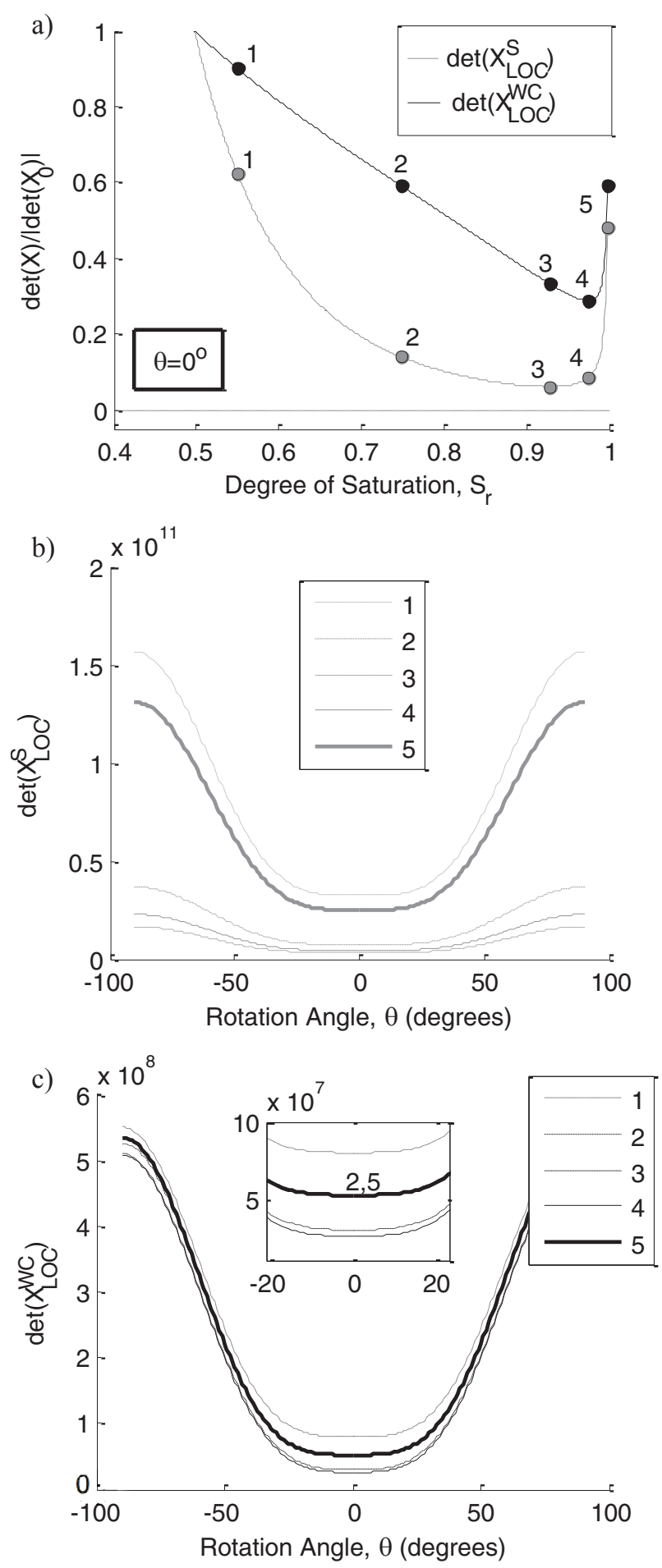

Figure 3. (a) Normalized controllability indices for suction controlled (light line) and water-content controlled wetting (dark line) for potential strain localization along horizontal bands, plotted against degree of saturation, (b) variation of loss of controllability index against shearing under suction control for a range of band angles, and (c) variation of loss of controllability index against shearing under water content control for a range of band angles.
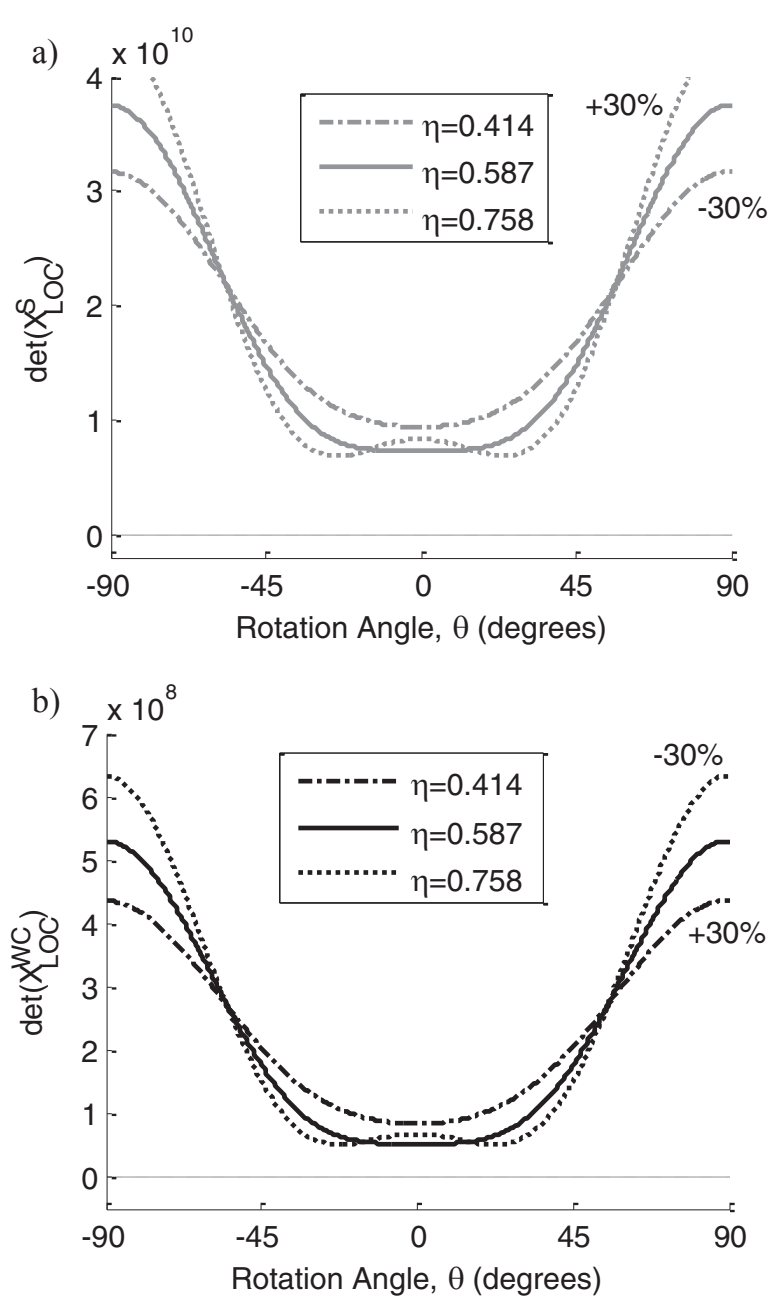

Figure 4. Controllability criteria for strain localization under suction-controlled (a) and water content-controlled wetting (b) plotted against band angle. Results are shown for stress ratios approximately corresponding with the oedometric wetting path at $S_{r}=75 \%$ (solid lines), $30 \%$ higher stress ratio (dashed lines) and $30 \%$ lower stress ratio (dotted lines).

\subsection{Influence of suction-induced hardening}

In the previous work on homogenous modes of instability [5], a parametric analysis was performed to study the role of the suction-hardening parameter, $r_{s w}$ on the stability upon wetting. It was found that increased values of this parameter made it possible for a loss of controllability to occur under water content control (injection).

The same type of analysis can also be performed to assess the potential of strain localization in materials with decreased suction-hardening during saturation. Figure 5 shows that indeed, higher values of $r_{s w}$ reduce the magnitude of both indices, (3) and (4). In fact, for the case of water injection, the index reaches zero, with localization at $\theta=0^{\circ}$ being predicted as the first potential loss of controllability (i.e., compaction banding). With higher suction-hardening values, the range of localization angles increases for the considered degree of saturation, $S_{r}=75 \%$. Hypothetically, a shear perturbation under any of the inclinations within the range highlighted in Figure $5 \mathrm{~b}$ would not be controllable during that point of the wetting path, for $r_{s w}=11$. 
Figure 6 compares water content control indices at this higher value of suction-hardening for different band angles with the index for homogenous instability used by [5]. At this value of $r_{s w}$, the first strain localization index to vanish is associated with localized deformation along a horizontal band (i.e., $\theta=0^{\circ}$ or compaction banding). This condition is met when also the stability of wetting paths under homogeneous oedometric deformation is lost. This result suggests that prior to a loss of controllability, the sample remains homogenous, but once controllability against water-content control is lost, homogeneity can no longer be guaranteed.
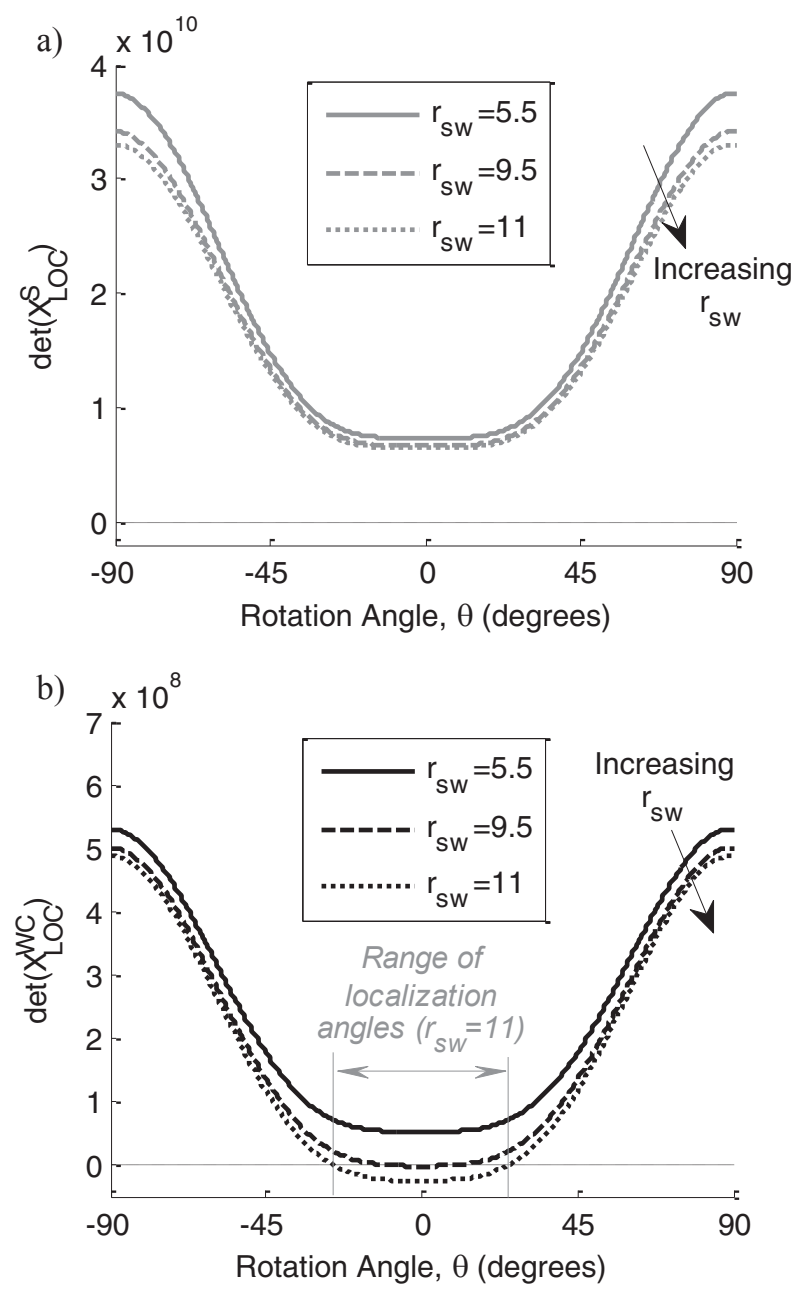

Figure 5. Controllability criteria for localization under suctioncontrolled wetting (a) and water content-controlled wetting (b) plotted against the angle of potential localization, for increasing suction-hardening.

\subsection{Influence of non-associativity}

Another material property that can influence the stability of soils is the degree of non-associativity. For instance, it is known that differences in the yield surface and plastic potential within an elastoplastic modelling context are needed to capture static liquefaction in sands $[16,17]$. Here the original wetting path simulation $\left(r_{s w}=5.5\right)$ is repeated for different degrees of non-associativity. The stress ratio at the peak of the surface shape $\left(M_{c}\right)$ is given different values for the yield surface $\left(M_{c f}\right)$ and plastic potential $\left(M_{c g}\right)$. Specifically, the latter, which is calibrated to match the critical state failure line, remains unchanged (i.e., $M_{c g}=M_{c}$ ), while $M_{c f}$ is taken as a percentage of $M_{c g}$. The peak stress values in extension ( $M_{e f}$ and $M_{e g}$, respectively) are maintained at around $80 \%$ of those in compression, and the remaining shape parameters are unchanged.

Figure 7 shows the effect of the introduction of nonassociativity. Not only does the shape of the localization profile change to favour strain localization along inclined band angles for increased non-associativity, but the water content control index (4) is actually reduced to zero or negative values at $75 \%$ degree of saturation. This indicates that non-associative materials can be prone to localization under water injection. In variance with the effect of increased values of suction-hardening (Figure $5 b)$, controllability is not lost for stress perturbations at $\theta$ $=0^{\circ}$, but it is met for a range of band angles (Figure 7b). Although loss of suction control was not computed in any of the considered simulations, also index (3) was found to be sensitive to a change of shape parameters, approaching lower values upon an increase of non-associativity.

Again, these results can be compared to the criterion for homogenous compaction under water content control (Figure 8). The results suggest that if the test remains homogenous, it should always be controllable and that horizontal compaction bands are not expected. However, increased non-associativity makes loss of controllability possible for stress perturbations acting along certain band inclinations. Since under experimental conditions, small deviations from the idealization of homogeneous material points are possible (e.g., heterogeneity, boundary effects) local deformation zones not compliant with oedometric conditions can take place within wetted samples. In other words, a range of band angles similar to those in Figure 7 may become possible, and localized zones of shear and/or compaction may appear while the oedometric test would still remain externally controllable. This finding is in agreement with recent observation of localization during wetting [7] of a highly non-associative, loose sand [12].

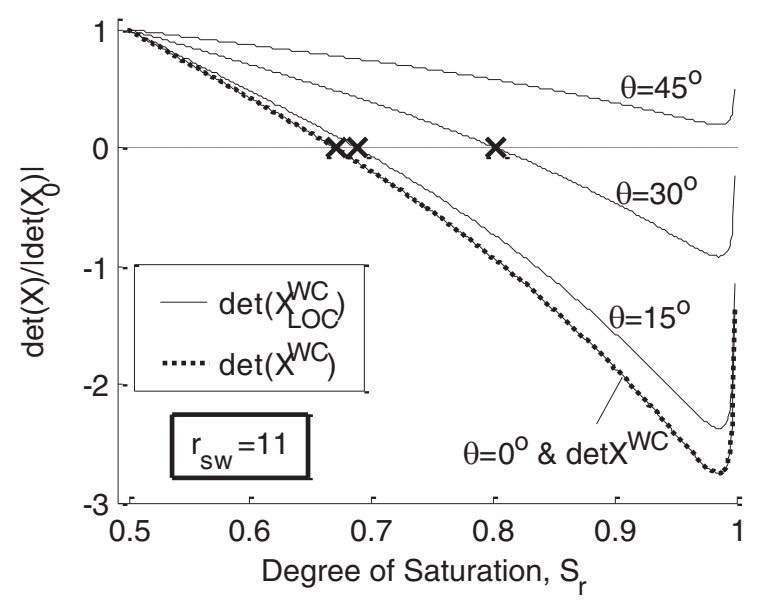

Figure 6. Comparison of the controllability indices computed for homogeneous (dotted line) and localized (solid line) wetting compaction for magnified values of suction-hardening $\left(r_{s w}=11\right)$. The symbol X marks the point at which the criteria are violated. 

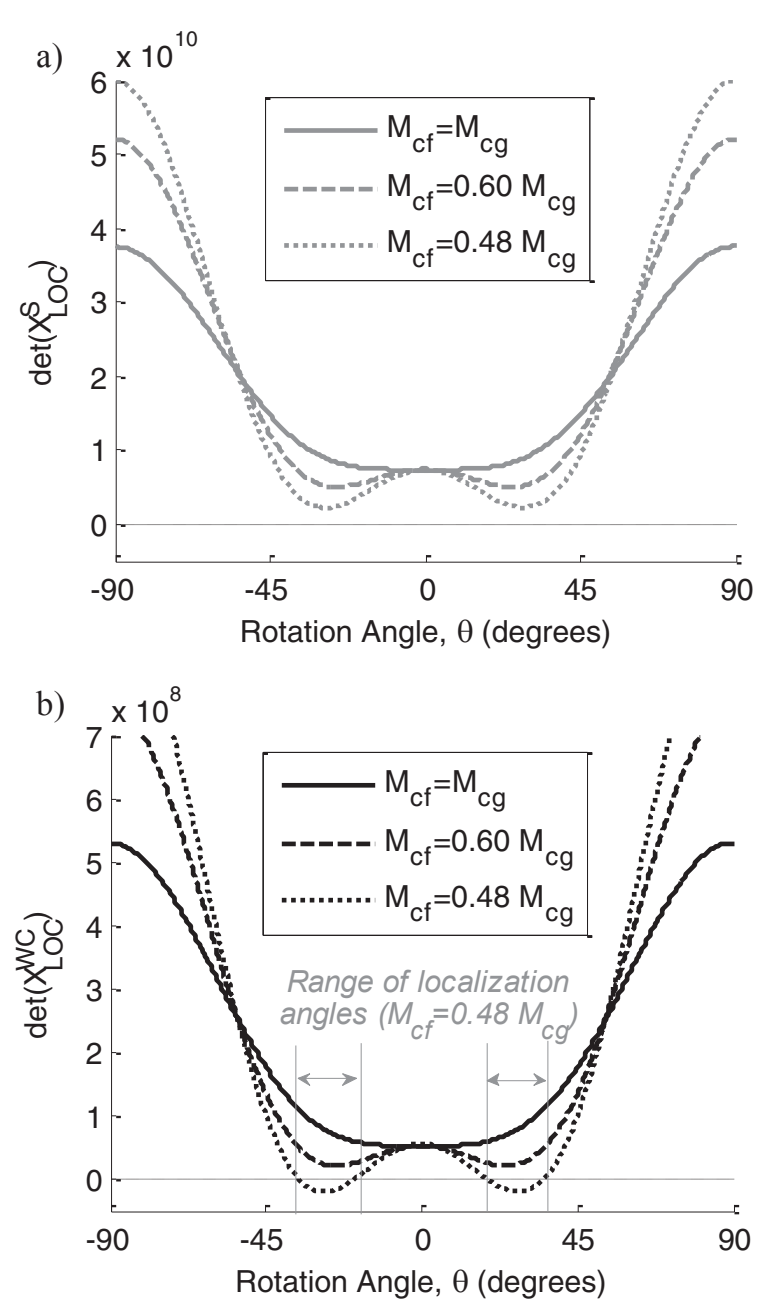

Figure 7. Effect of non-associativity on controllability criteria for (a) suction and (b) water content-controlled wetting at $r_{s w}=5.5$, plotted against the potential band angle.

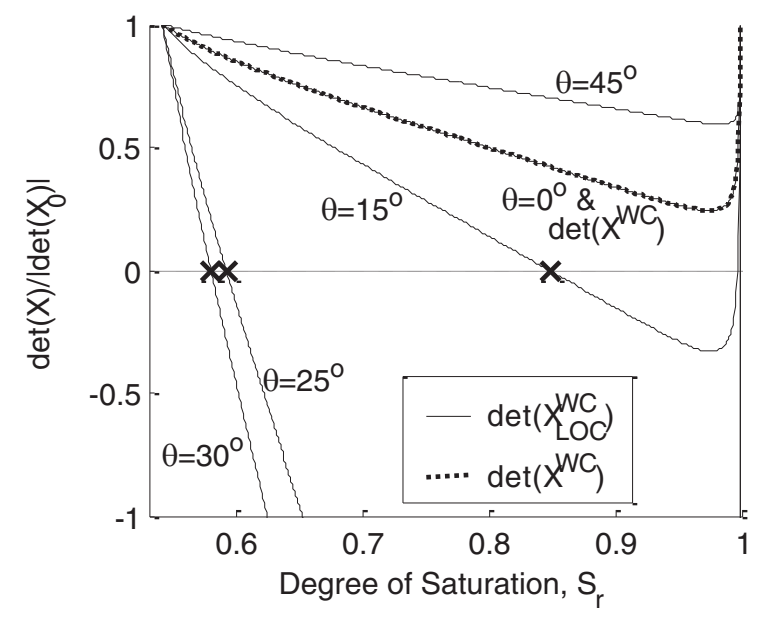

Figure 8. Comparison of water content control indices for homogeneous (dotted line) and localized (solid line) wetting compaction, for magnified non-associativity: $M_{c f}=0.48 M_{c g}$.

\section{Conclusions}

Conditions for loss of controllability against shearing were used to assess the potential for strain localization during wetting. Criteria were defined for saturation under suction removal and fluid volume injection. These criteria were computed for a range of band inclinations. While suction-controlled wetting has never found to cause any violations of the controllability criteria, different material properties did affect the potential for strain localization under water volume control. Variations in the stress ratio were found to change the range of band angles at which controllability indices reached a minimum, and increased values of the suction-hardening parameter were found to favour the formation of shear/compaction zones. For associated soils, such heterogeneities were found to be possible only at or after a loss of control for homogenous compaction. By contrast, it was found that an increase of the degree of non-associativity tended to promote shear and/or compaction bands even for degrees of saturation at which the homogenous compaction was still controllable. This suggests that small deviations from an ideal material point scheme (e.g., due to soil heterogeneity) may cause noticeable strain localization during wetting paths. These predictions are in agreement with recent experimental findings and can be used to gain a better understanding of the mechanics of wetting-induced deformations.

\section{References}

1. E. C. Lawton, R. J. Fragaszy, M. D. Hetherington, J. Geotech. Engrg. 118, 9 (1992)

2. G. A. Miller, K. K. Muraleetharan, Y. Y. Lim, Transport. Res. Rec. 1755 (2001)

3. K. D. Walsh, W. N. Houston, S. L. Houston, J. Geotech. Engrg. 119, 5 (1993)

4. S. L. Houston, J. Transp. Eng. 114, 6 (1988)

5. C. Mihalache, G. Buscarnera, J. Geotech. Geoenviron. Eng. 141, 2 (2014)

6. G. Buscarnera, R. Nova, Int. J. Numer. Anal. Meth. Geomech. 35 179-200 (2011)

7. J. F. Bruchon, J.-M. Pereira, M. Vandamme, N. Lenoir, P. Delage, M. Bornert, Granul. Matter 15, 6 (2013)

8. J. W. Rudnicki, J. R. Rice, J. Mech. Phys. Solids, 23, 371-394 (1975)

9. R. I. Borja, Comput. Methods Appl. Mech. Engrg. 193, 5301-5338 (2004)

10. S. Imposimato, R. Nova, Mechanics of CohesiveFrictional Materials 3, 65-87 (1998)

11. G. Buscarnera, C. di Prisco, Geotechnique Letters 1, 85-90 (2011)

12. C. Mihalache, G. Buscarnera, Int. J. Numer. Anal. Meth. Geomech. 38, 2 (2014)

13. G. Buscarnera, C. di Prisco, Int. J. Numer. Anal. Meth. Geomech. 36, 36-49 (2012)

14. K. A. Issen, J. W. Rudnicki, J. Geophys. Res. 105, B9 (2000)

15. F. Marinelli, G. Buscarnera, Int. J. Rock Mech. Min. 78, 240-252 (2015)

16. G. Buscarnera, A.J. Whittle, Can. Geotech. J. 49, 5, 499-511 (2012)

17. G. Buscarnera, A.J. Whittle, J. Geotech. Geoenviron. Eng. 139, 3, 420-432 (2013) 